\title{
Libertinaje y desborde: el exilio interior de Mario Levrero
}

\author{
Carlos BELLO RODRÍGUEZ \\ Universidad Complutense de Madrid
}

\begin{abstract}
RESUMEN
Dentro del marco de represión y censura impuesto por la dictadura uruguaya (1973-1985), la producción de Mario Levrero vivió su particular punto de fuga. Su "trilogía involuntaria" conformada por La ciudad, El lugar y París (escritas entre 1966 y 1970)- daba paso en 1973, en paralelo al estallido totalitario, a la escritura de Nick Carter se divierte mientras el lector es asesinado y yo agonizo, nouvelle paródica caracterizada por la irrupción del humor como instrumento, como útil, como auxiliadora terapia levreriana.
\end{abstract}

Palabras clave: exilio interior, insilio, dictadura uruguaya, autocensura, literatura fantástica, literatura policíaca.

Overflow: Mario Levrero's isolation in the Uruguayan dictatorship

\begin{abstract}
Framed by the context of repression and censorship imposed by the Uruguayan dictatorship (1973-1985), the production of Mario Levrero had its particular leaking point. His 'accidental trilogy' -formed by La ciudad, El lugar and Paris (written between 1966 and 1970)- gave place to the writing of Nick Carter se divierte mientras el lector es asesinado $y$ yo agonizo, parodic nouvelle that is characterized by the irruption of humor as a useful therapy.
\end{abstract}

Keywords: interior exile, Uruguayan dictatorship, self-censorship, fantastic fiction, crime fiction.

SUMARIO: 1. Mario Levrero y el afuera. 2. Libertinaje y desborde. Nick Carter se divierte mientras el lector es asesinado y yo agonizo. 3. A modo de conclusión.

\section{Mario Levrero y el afuera}

El desapegado y hermético y fóbico Jorge Mario Varlotta Levrero, el hombre que rara vez salía de su casa -qué decir de su barrio-, el parapsicólogo y telépata, el humorista que difícilmente concedía entrevistas $-\mathrm{y}$ si las concedía, se afanaba en boicotear hablando en voz baja-, el defensor de la hipnosis como procedimiento terapéutico y de las percepciones extrasensoriales, el hombre que no abría la puerta si el llamante le transmitía algún tipo de energía negativa, el hombre que para los tratos con la realidad se llamaba Jorge Varlotta y que para la literatura se llamaba Mario Levrero, el excepcional escritor que permea como pocos la relación 
persona/personaje, el excepcional orillado, el excepcional que Mario Levrero fue se declaraba -si es que alguien se atrevía en algún momento a molestarlo preguntándole por lo político- "monárquico".

Acto seguido -cuenta su amigo Edgardo Lizasoaín, Lizán- apoyaba su declaración presentando "con sorna y orgullo travieso" una "no muy comprobable y forzada relación de parentesco con cierto conde europeo", establecida de acuerdo a "un brumoso árbol genealógico que le había insumido algún tiempo estudiar y desentrañar". Lo explica Lizasoaín en una entrevista realizada por Jesús Montoya Juárez (2013: 30) para el estudio de campo que daría origen a su "Mario Levrero para armar", primer capítulo de su libro homónimo y que, hasta el momento, surge como la única aproximación biográfica existente a la figura del uruguayo.

El laborioso trabajo de Montoya Juárez recoge, como "un recuerdo común en varios de los informantes entrevistados", el rechazo de Levrero a intervenir en las conversaciones que abordaban problemas de la actualidad política o su enojo cuando era interpelado a este propósito. Supone Carla Varlotta ${ }^{1}$ que en algún momento debió sufrir una decepción que hizo que este abandonase la militancia y apenas la refiriera en adelante (30).

Esa "militancia" a la que Carla Varlotta se refiere -sorprendente a la vista del Levrero alejado de la cosa pública que posteriormente sería- es el mismo hecho biográfico sobre el que varios de los amigos o familiares entrevistados por Montoya Juárez coinciden en detenerse: la afiliación del joven Varlotta, a comienzos de la década de 1960, a la Unión de Juventudes Comunistas (UJC) y su implicación, como la del resto de sus amistades de la época, en la participación política de los tempranos 1960 montevideanos (27-29).

En la trayectoria, vital y productiva, de Levrero esto precede a un momento clave -un parto de sí mismo, dirá él- a partir del cual abordará la escritura de sus primeras novelas y cuentos - La ciudad, escrita en 1966 y publicada en 1970; El lugar, escrita en 1969 y publicada en 1982; París, escrita en 1970 y publicada en 1979; junto con el libro de relatos La máquina de pensar en Gladys, escritos entre 1966 y 1970 y publicados en este último año ${ }^{2}$-, además de innumerables y desopilantes colaboraciones para revistas humorísticas de la época.

\footnotetext{
${ }^{1}$ Hija biológica de Mario Levrero, fruto de su unión con María Lina.

${ }^{2}$ La información sobre los años de producción se recoge de los paratextos entregados por el propio Mario Levrero, que acostumbra a finalizar sus ficciones haciendo referencia al año y, normalmente, a la ciudad en que fueron escritas. Cuando esos paratextos no existen es el caso de París-, recurro a la fecha de escritura revelada oralmente por Levrero. " $E l$ Lugar, de 1969, forma parte de lo que podría llamarse una 'trilogía involuntaria'. La misma se inicia con La Ciudad, de 1966, y culmina con París, de 1970. En las tres domina la búsqueda más o menos inconsciente de una ciudad", le expresará a Elvio Gandolfo (Olivera, 2008: 175).
} 
El tiempo abierto en 1973, y al que Mabel Moraña se ha referido en extenso en Memorias de la generación fantasma, constituirá en Levrero -a decir de Carla Varlotta- un momento histórico vivido con aprehensión que a la postre "agravó y cronificó muchas de las fobias y los miedos de Levrero, entre ellos una cierta agorafobia". Según recordaba María Lina, la madre de Carla, por aquel tiempo este "cerró las persianas, no atendía al timbre ni al teléfono" (Montoya Juárez, 2013: 47).

En 1996, y preguntado sobre la cuestión por Hugo Verani, Levrero abundó en cómo afectó la dictadura a su escritura. El texto forma hoy parte de la compilación realizada por Elvio Gandolfo para su tomo de entrevistas a Mario Levrero:

La década larga de dictadura, que pasé íntegramente en Uruguay, me afectó de distintas maneras y en todos los órdenes de la vida, incluyendo la literatura; no voy a describir ahora sentimientos y anécdotas muy diferentes de los que habrá conocido la gran mayoría de la población. La censura no tenía mucho que hacer con lo que yo pudiera escribir, pero sí la autocensura paranoica (razonablemente paranoica): "que no se vaya a encontrar en mi casa una hoja de papel con algo escrito que pueda ser interpretado como...". Eso es terrible. (Gandolfo, 2013: 123)

Acto seguido, Levrero aprovechaba la ocasión para arremeter contra la crítica literaria en tanto que, como él siempre defendería, los críticos "ejercen una forma de poder destructivo (análisis implica destrucción)". Impetuosamente huidizo de cualquier relación analítica entre su obra y cualquier cosa que pudiera oler a política, a Levrero le importunaba especialmente la aparición de comentarios como el que, en 1988, el crítico Juan Carlos Mondragón deslizó sobre su denominada "trilogía involuntaria" (La ciudad, El lugar y París). Aunque sus novelas, decía Mondragón, se sitúan "en zonas de la literatura en apariencia descontextualizadas de los tiempos que corren, [...] Levrero proporciona un 'diagnóstico' inquietante, sus textos no compiten ni con el testimonio ni con la historia, pero se instalan de manera inconfundible en la superestructura de nuestra sociedad, y lo hacen de forma diferente, heterodoxa" (Olivera, 2008: 455).

En esa misma entrevista con Verani, Levrero llegaba a expresar que "hay algo maligno en inventar referencias (a la dictadura o a cualquier cosa) donde no las hay". Y añadía: "las referencias conscientes a ese triste período son escasas y concretas", pero entre ellas se encuentra, decía Levrero, "un breve pasaje de Nick Carter" (Gandolfo, 2013: 123).

El crítico como enemigo, en tanto que hermeneuta empeñado en encontrar referencias y establecer categorizaciones ${ }^{3}$, esa imagen levantada por un Levrero al

${ }^{3}$ La insistencia de Mario Levrero en defender su producción como "literatura realista", por ejemplo, se repetirá en sus conversaciones de forma más que reiterada. Con pequeñas variaciones, afirmaciones como "lo que escribo es realismo puro [...] son todas cosas que de 
que hasta los prólogos parecen molestarle -"da la impresión de que Levrero es partidario de que no se hable de nada", escribirá Ignacio Echevarría precisamente en un prólogo (2008b: 8)-, tuvo su contraparte en un momento crítico que Levrero cultivaría en los años 1980 y del que, cuenta Montoya Juárez, enseguida se arrepintió. Lo más sorprendente de aquellos trabajos, explica el profesor, se encuentra en esta lectura que el crítico Levrero haría en El País Cultural de la narrativa de César Aira, entendiendo como un acto político el hecho de que el escritor argentino abandone en sus ficciones la temática política. Mario Levrero reflexiona Montoya Juárez-

lee en la ironía, el abandono de la temática política y la renuncia a hacerse cargo de la historia reciente de novelas como La hija de Kheops, La perla del emperador o Una novela china, un síntoma del trauma generacional que no puede aún confrontar los hechos terroríficos vividos. La lejanía respecto de lo político es observada a la vez como un hecho político indiscutible por Levrero. Pese a ello siempre se resistió a que buena parte de su propia obra fuera leída desde ese planteamiento. (2013: 53)

Lo central, claro, de estas cuestiones biográficas, su punto de cruce, es que pese a la negativa declarada por el autor a que se establezcan vinculaciones contextuales con su obra, 1973 coincide con un salto, con una importante muda en su narrativa ${ }^{4}$.

Jean-Paul Sartre -en Qué es la literatura (1948)- explicaría que este cambio podría, ciertamente, mirarse como derivado de lo contextual en tanto que, pese a su aparente negativa por estarlo, el escritor se 'sitúa', como cualquiera de nosotros, en un espacio y en un tiempo histórico del que no tiene modo de evadirse. En aquel momento y en aquel lugar, en un paisaje donde el crimen, la tortura y la represión no pueden cegarse -no son éstos, además, céfiros que toquen precisamente suave, sin alterar- la dictadura le afecta a Levrero, como a cualquier uruguayo de ese

alguna manera me pasaron, en algún plano interior", a Eduardo Berti y Jorge Warley (Gandolfo, 2013:27); "Yo a Kafka lo considero un realista", a Cristina Siscar (40); "la gente se sitúa en un lado de la realidad y me ponen a mí en el otro, como si lo que yo escribo no fuera real. Entonces aparecen los dueños de la realidad, que siempre son los críticos", a Juan Antonio Bruno, Alberto Galione y Jorge Bonino (138), se repiten casi a cada conversación con él. En 1992, Pablo Rocca acabaría proponiéndole para sus primeras novelas -sin duda encuadradas, según argumentará Jorge Olivera, en el "fantástico moderno"- el término de "realismo introspectivo", que Levrero celebró como una "expresión sumamente adecuada" (De Rosso, 2013: 107).

${ }^{4}$ Aunque exceda los límites de este artículo, creo importante hacer notar que el fin de la dictadura también coincidirá con la entrada en otra etapa narrativa. Recién restituida la democracia, 1986 será el año en que Levrero arranque, desde Buenos Aires, una producción inédita en él hasta ese momento: la diarista y autoficcional. 
momento, en tanto que le abraza estrechamente. Y lo hace, apostillaría Sartre, aunque decidiera no mirar. "Aunque nos mantuviéramos mudos y quietos como una piedra, nuestra misma pasividad sería una acción. Quien consagrara su vida a hacer novelas sobre los hititas tomaría posición por esta abstención misma. El escritor tiene una situación en su época; cada palabra suya repercute. Y cada silencio también" (Sartre, 1967:10).

Así, mientras algunos de sus amigos son encarcelados, otros se exilian, otros sufren el encarcelamiento de sus familiares, y con el propio Levrero viéndose obligado a ocultar a los hijos de su amiga Liliana Guemberena por el temor a que los militares entren a su casa (Montoya Juárez, 2013:46), Levrero reacciona exiliándose, propiamente, en sí mismo ${ }^{5}$. A decir de sus amigos, durante esa época Levrero "debió sufrir episodios depresivos" y "se sometió a diferentes tipos de terapia". Serían tiempos, además, en los que Levrero profundizaría en sus estudios parapsicológicos y en los que, según el testimonio de su amiga Perla Domínguez, conocería a un sacerdote con el que ocupará innumerables tardes jugando al ajedrez (47).

Pero el Levrero alejado, semiinvisible, maniático y anacoreta, más que un "indiferente moral", un apartado por decisión propia, uno de aquellos hombres "sin fe ni interés por su destino" que Juan Carlos Onetti referiría en su epígrafe a Tierra de nadie, seguramente sea un resultado procesal. En Del centro a la periferia. Encuentros y transgresiones en la literatura uruguaya, Fernando Aínsa explica que, dado que "siempre texto y contexto se explican y complementan en el seno de la historia" (2002: 13), la existencia de una producción literaria que posteriormente acostumbrará a "proyectar alegorías y mitos desde la realidad" y a "trascender lo cotidiano por la desmesura y el absurdo" (2002: 131) se relaciona indefectiblemente con la existencia del período dictatorial: "[a la narrativa actual] ha contribuido no solo la tradición literaria inaugurada por El pozo de Juan Carlos Onetti y Por los tiempos de Clemente Colling de Felisberto Hernández, sino la propia historia reciente del país" (2002: 138). Y explica que "la sensación de vivir un exilio interior conducía en forma irremediable a una visión marginal y sesgada de una realidad que no podía ser abordada frontalmente. No es extraño, entonces, que al restablecerse la normalidad democrática [...] la escritura ya estuviera irremediablemente marcada por ese enfoque y postura" (2002: 139).

${ }^{5}$ Como es bien sabido, frente la diáspora siempre cabe el insilio. Pero cuando éste se produce, señala Mabel Moraña, cuando el escritor permanece en Uruguay "sin optar, como sucede a veces, por el silencio", "su producción se desarrolla como un riesgoso equilibrio entre su conciencia, su ética, su axiología, y los dictados del sistema dominante, que reglamenta y controla todas y cada una de las formas de expresión. La obra literaria surge entonces como manifestación de esa coyuntura conflictual” (Moraña, 1988: 20). 
El escritor marginal, el excéntrico (literalmente, "fuera del centro" o "que tiene un centro diferente") que fue Mario Levrero podría, ciertamente, tener relación entonces con esa "invitación al exilio interior, a replegarse sobre sí mismo, a salirse del gran cauce, a hacerse a un lado" (2002: 133) de la que habla Aínsa: ¿Hasta dónde la marginación es una vocación deliberadamente asumida o (por el contrario) es el resultado de un sistema que expulsa hacia sus bordes, que excluye a quienes no aceptan las reglas y convenciones de la corriente mayoritaria, exclusión que ha sido flagrante en el período de la dictadura? (2002: 132).

Empujando hacia el desasosiego y el desencanto, es la propia estructura social dice el hispanouruguayo- la que "empuja hacia las orillas del sistema, a una forma de exilio interior a las conciencias desdichadas (Hegel) y a los poseedores de virtudes incomprendidas por las mayorías" (2002: 132).

Seguramente sean esas "virtudes incomprendidas" las que, texto tras texto, pueden llevar a toparse junto al nombre de Levrero el inquietante adjetivo de "inclasificable", esas peculiaridades que, junto a su desidia por la cuestión de ser publicado o no y su negativa a las tareas de la promoción y la visibilidad, estén en la base de una sorprendente ausencia de reconocimiento a una obra sin duda luminosa pero que, explica Jorge Olivera, era vista como "la de un autor situado en los márgenes de la literatura establecida. Hasta bien entrados los años ochenta en las librerías de Montevideo y Buenos Aires era muy difícil encontrar ejemplares de la obra de Levrero" (Olivera, 2008: 7).

Sea como fuere, lo cierto es que en 1973, durante aquellos meses en que irrumpe la dictadura, la narrativa de Levrero inaugura una nueva forma expresiva, recala en un nuevo estadio, cambia, muda.

Desde sus anteriores paisajes de la pesadilla, del discurrir flâneur por lo angustioso y lo incomprensible -hasta ese momento, los narradores de sus novelas y

${ }^{6}$ Aunque de mirada oblicua y espíritu ciertamente libre, los textos de Mario Levrero se mueven - cubriendo una doble etapa- dentro del territorio de lo que concede en llamarse "literatura fantástica" y, posteriormente, "literatura autoficcional". Más concretamente, y siguiendo las cuestiones aportadas por Ana María Barrenechea, Enriqueta Morillas Ventura y Rosalba Campra, Jorge Olivera circunscribe aquellas primeras obras al territorio del "fantástico moderno". A pesar de ello, no han sido pocas las veces que -recibiéndolo ya como un alarmante ritornello- el adjetivo "inclasificable" me asaltó desde diferentes trabajos críticos. Consta, por ejemplo, en los textos de Fernando Aínsa - "inclasificable explorador de géneros y sub-géneros diversos" (1993: 47)-, de Ignacio Echevarría -“una obra tan difícilmente clasificable como la de Mario Levrero" (2008b: 7)-, de Hugo Verani "su atracción por las zonas oníricas y las penumbras que envuelven los procesos mentales genera una modalidad expresiva inclasificable"- (Gandolfo, 2013: 39) o de Luciana Martínez -"la inclasificable obra del escritor uruguayo" (165)-. Entiendo que esto debe tener que ver con la reiterada negativa, expresada innumerables veces, por Levrero a ser encasillado como autor de "literatura fantástica" o "ciencia ficción". 
cuentos siempre paseaban un entorno insoportable que no pueden comprender y para el que nadie facilita las respuestas-, Levrero escribe dos textos que, apoyados ahora en el uso de la parodia, del humor y del narrador esquizoide, por un lado, y en el establecimiento de la contradicción discursiva como modelo de avance narrativo, por otro, establecen una clausura: la de sus espacios de túnel y laberinto, de ciudades que atrapan, de recorridos por mundos y submundos opresivos e inquietantes.

La escritura de Nick Carter se divierte mientras el lector es asesinado ${ }^{7}$ convierte su producción en lúdica, clausura aquellas ficciones oscuras, angustiosas y laceradas que -ésta es, siguiendo su pista de la "autocensura paranoica", mi lecturaLevrero podría haber dejado de practicar en tanto que susceptibles de ser tomadas por alegóricas.

Afirma Jorge Olivera que en aquellas tres novelas kafkianas de Levrero "se sospecha" la existencia de "una tendencia que anticipa lo que serían posteriormente las formas de expresión de la realidad bajo censura [en el Uruguay de los 1970 y 1980]" (2008: 158). Ciertamente, y tal como historian Mabel Moraña, Fernando Aínsa o Teresa Porzecanski, la represión dictatorial propiciará durante los años siguientes una literatura del enmascaramiento vehiculado a través de lo fantástico, unas "formas del decir elusivo", a decir de Porzecanski que, naturalmente, incidían en un uso recurrente de la alegoría que, a su vez, ponía en marcha a un sistema censor afanado en detectarlas ${ }^{8}$.

${ }^{7}$ También la de Caza de conejos, escrita en ese mismo año y que se configura en la sucesión de escenas narrativas mínimas donde la historia se dice y se desdice, donde los episodios se espejean y contraponen. El acercamiento a esta obra formará parte de un próximo artículo, aún en preparación.

${ }^{8}$ Según refiere Jorge Olivera, el primer editor de El lugar, temeroso de que la escena pudiera ser interpretada, instó a Levrero a que cercenara un pasaje que el autor concedería sin problemas omitir. "Había una escena donde aparecía un encapuchado, y como podía interpretarse con un contenido político me pidieron permiso para cortarla. Como la novela fue publicada en plena dictadura, acepté", declararía en 1988 Levrero a Carlos María Domínguez (cit. Olivera, 2008: 245). El evitado pasaje, ya recuperado en ediciones posteriores, relataba: "Me introduje en la única habitación cuya puerta pude abrir. Un grupo de hombres, cuatro de ellos desnudos y un quinto encapuchado, azotaba a una mujer que tenía las muñecas y los tobillos unidos a la pared por cadenas metálicas. Los hombres tenían acentuados rasgos mongólicos. Intenté huir pero me dieron alcance en el corredor. Silenciosamente me llevaron de vuelta a la pieza y colocaron el látigo en mis manos. Me enfrentaron a la mujer, que sangraba y balanceaba su cabeza pesadamente sobre los hombros, y gemía. Me golpearon las costillas y descargué un latigazo sobre la mujer; les pareció demasiado suave y volvieron a golpearme. Tomé el látigo del revés, por donde terminaba la parte rígida, y comencé a dar golpes con el mango, en todas direcciones. El encapuchado exhibió un revólver, pero yo había conseguido alcanzar la puerta; hacia el final 
En medio de la brutalidad y lo nefando, entre la impudicia y la violencia, la literatura de Levrero se hace más libre, se abre, se libertina, se desborda en lo que, sorprendentemente, en realidad no deja de ser un repliegue ante lo contextual, una forma de cementar las fisuras que pudieran dar origen a interpretaciones críticas indeseadas, a desencaminados análisis, a 'diagnósticos'.

\section{Libertinaje y desborde. Nick Carter se divierte mientras el lector es asesinado y yo agonizo}

Cuando, en 1985 y por motivos estrictamente económicos -valdría incluso decir de pura subsistencia-, Mario Levrero cambia su residencia a Buenos Aires, se encanta, se maravilla en el paseo por una ciudad ante la que, al "ver la vida bullendo, el movimiento", detalla Levrero, se le "llenaban los ojos de lágrimas". Buenos Aires, dice, "contrastaba por completo con la imagen que mi propia depresión me había dado de Montevideo: el horizonte cerrado, las esperanzas fracasadas" (Gandolfo, 2013: 33). Pese al valor, siempre juicioso, que debe atribuírsele a lo declarado en entrevistas por Levrero, el autor ha insistido en varios lugares en señalar el valor distractor o terapéutico con el que para él cuentan las ficciones policiales": "Cuando no estoy distraído -esas etapas de depresión que paso leyendo novelas policiales- y reparo en el entorno y en mí mismo, todo me parece muy extraño" (2013: 191). Se tratan éstas, a decir de Levrero, de ficciones útiles "para paliar estados de nerviosismo o malestar físico" durante "períodos de depresión, estados de ánimo desagradables" (Montoya Juárez, 2013: 115, cit. Olivera y Acosta, 2006).

Pero Nick Carter se divierte mientras el lector es asesinado y yo agonizo escrita en 1973 y publicada por primera vez en 1974 como folletín por entregas- no es una nouvelle policial al uso, sino un excéntrico regocijo imaginativo presentado como disparatada parodia del género donde Levrero abre una brecha lúdica, se carcajea de las convenciones narrativas, subvierte géneros populares, dinamita el verosímil y -firmándola no como Mario Levrero sino como Jorge Varlottapresenta por vez primera en su narrativa un narrador esquizoide, dividido entre el uso de la primera y de la tercera persona que también se dirige a sí mismo o interpela, en clave existencialista, al lector. Al escribirla Levrero rompe con la pesadilla, desborda su etapa del discurrir por la asfixia kafkiana y sitúa su novelística -recordemos el valor liberador que para la teoría freudiana tiene la risa-

del corredor sentí que una bala me rozaba el brazo sin llegar a herirme" (Levrero, 2008: 146-147).

9 Ignacio Echevarría amplía los márgenes de esta consideración afirmando que en general la literatura, la escritura en sí, el acto de escribir "cumple para Levrero una función auxiliadora" (2008a: 99). 
en la vía expresiva de sus colaboraciones para las revistas humorísticas y que, retomando las palabras escritas un año antes por Ángel Rama, se concretaría ya en un uso, propiamente sin bridas, de lo que éste denominaría "la imaginación al poder" (1972: 101) ${ }^{10}$. A su juicio, algunas de las páginas escritas por Levrero y Peri Rossi en los últimos 1960 conformaban "los ejemplos más libres de imaginación que hayan conocido las letras uruguayas" (244), y aunque "si bien Nick Carter", afirma Montoya Juárez, "no estuvo en la mira de Ángel Rama cuando en 1972 motejó de libertinaje imaginativo su proceder literario, creo que particularmente esta nouvelle es quizás el texto donde mejor encuentra acomodo esta expresión" (Montoya Juárez, 2013: 121).

Preguntado por Elvio Gandolfo sobre la gestación de esta juguetona parodia, encuadrada dentro del género neopolicial y que tanto pareciera disfrutar fagocitando modelos de la cultura popular, Levrero afirmará que "[por aquel tiempo] tenía unas ganas confusas de escribir algo policial, pero en tono surrealista. Pensaba que tenía que ser con un detective borracho, drogado o loco, alguien que se encontrara de una manera un poco mágica con la realidad. Con Nick Carter me divertí ferozmente" (Gandolfo, 2013: 19).

El texto, que a decir de Jorge Olivera, "revela, no solamente situaciones absurdas y rayanas en lo insólito, sino también una fina dosis de humor" (2008: 167), mezcla "ficción pulp, tira cómica y delirio onírico, con algunos delicados toques kafkianos" (Echevarría, 2008b: 9) y, en palabras de Pablo Silva Olazábal, "se desbarranca en el todo vale, o todo pasa porque si" (2008: 33), no llevó, en cambio, la firma de Mario Levrero. Lo explica en una entrevista con Carlos María Domínguez:

Nick Carter apareció como algo distinto. No lo vi en la misma línea que lo demás, no lo asimilaba al resto de la obra de Levrero, y en ese momento me parecía algo inferior. Mientras lo escribía pensé que no se lo podía mostrar a nadie. Estaba volcando mucha cosa psicoanalítica personal y fantasías de todo tipo, principalmente eróticas. Intenté buscar otro seudónimo pero al final aquello fue una decisión de los editores. Me enteré de que estaba como Jorge Varlotta cuando salió el libro. (Gandolfo, 2013: 50)

${ }^{10}$ Agrupando bajo este rótulo a Mario Levrero, Cristina Peri Rossi, Teresa Porzekansky, Mercedes Rein, Gley Eyherabide y Jorge Onetti, Rama se refería a una nueva hornada de escritores que, lejos de merecer por ello el aplauso, mostraban en Uruguay una "imaginación de libertad excedida y de carencia de fiscalización de lo real" en vez de intentar, decía, “encauzar la realidad por un nuevo derrotero" (Rama, 1972: 244-245). En lo que a Levrero respecta, el crítico se refería, además de a La ciudad (publicada en 1970), al cuento "Gelatina" (publicado por primera vez en 1966 y en el que una masa informe "se come" la ciudad). 
Pese a que el autor ha referido que, en el momento de escribir Nick Carter se divierte mientras el lector es asesinado y yo agonizo, "no conocía para nada" (Gandolfo, 2013: 19) al homónimo personaje que en 1886 creara John Russell Coryell, es importante notar, como historia Ezequiel de Rosso, que Nick Carter era un conocido personaje folletinesco.

La popularidad de Nick Carter tuvo lugar entre fines del siglo XIX y 1920, es decir, aproximadamente en el mismo momento en que Sherlock Holmes gana su lugar en la escena literaria británica. Así leído, Nick Carter es la contracara norteamericana y bastarda de la historia oficial del género. Por lo demás, el ayudante de Carter en la novela de Levrero se llama Tinker, que es el nombre del ayudante de Sexton Blake (uno de los detectives ingleses más populares del mismo período que Nick Carter). Se trama así un conjunto de referencias (al que habría que agregar la aparición de un doctor Watson como criminal) que aluden a una tradición marginal y profundamente folletinesca, como si se declarara que el interés de la novela policial no está en Holmes, sino en sus epígonos. (De Rosso, 2013: 156)

El intuitivo y esotérico Nick Carter de Levrero, haciendo explosionar, explica Montoya Juárez, las reglas clásicas sobre las que Borges y Bioy establecían el "verdadero" policial, "emplea el sueño, la hipnosis y toda una serie de instrumentos parapsicológicos en sus investigaciones" y "la trama detectivesca se diluye, deja de importar en ocasiones, o se fractura en diferentes tiempos y planos o dimensiones de realidad" (Montoya Juárez, 2013: 113). Además, el narrador se divide, se fragmenta, está violentado y fuera de quicio, interpreta voces nuevas que indistintamente usan la primera persona o la tercera o la segunda:

Han pasado muchos años [es Nick Carter quien habla]. Mi abuelo ha muerto hace tiempo. Pero en homenaje a su memoria juro que, una vez finalizada la investigación en el Castillo, iré a Escocia a estudiar el problema del volcán.

Con los primeros acordes del "Vals del Emperador", Nick Carter aparece en lo alto de la escalinata de mármol. Se mueve levemente al ritmo del vals. Viste un impresionante traje de gala, del cual se va despojando mientras baja los escalones. (Levrero, 2012: 39-40)

Allá vas, diciéndote: "Aquí viene Nick Carter, el detective más famoso del mundo, a resolver un enigma". Pero en el fondo de tu almita sabes que no es cierto. El enigma eres tú, Nick Carter, el único enigma verdadero que nunca has podido resolver, el enigma de tu vida vacía, de tu verdadera identidad. ¿Cuánto dinero has entregado a los periódicos para que hinchen tus hazañas? (71).

Y tú, lector, que te apiadas del vacío de Nick Carter, ¿qué me puedes decir de ti mismo? De tu enigma, de tu identidad. ¿No te has dado cuenta de que también a ti te han asesinado? A ti también te han clavado un cuchillo en la espalda el día mismo en que naciste. Pero en tu ceguera le llamas vida a tu vida, a eso que 
arrastras, como tantos lectores, infectando el mundo. Todavía no ha nacido el detective que investigue tu muerte, lector. Nunca serás vengado, anónimo gusano. No eres mejor que Nick Carter, ni que yo. (71-72)

Pero, identitariamente disociada, las peripecias que esta voz narra también aparecen desdobladas y simultáneas, lo que llevará al personaje a convertirse en mero observador de sí mismo - se aficiona a seguir sus propias aventuras- en la televisión: "Se aproxima la hora del programa televisivo de LAS AVENTURAS DE NICK CARTER. Enciendo el televisor colocado frente al sofá, comprado especialmente para seguir paso a paso esta serial maravillosa" (Levrero, 2012: 41), o a contemplar su imagen especular tomando vida propia mientras él, atónito, no puede por más que observarla:

Mi imagen había regresado al espejo, acompañada de la hija menor de Lord Ponsonby. Ambas imágenes estaban desnudas y se acariciaban impúdicamente. Mi imagen se había acercado todo lo posible a la superficie del espejo y exageraba sus obscenidades. Si el Lord se daba vuelta, yo estaba perdido. La hija del Lord era una niña; apenas diez u once años. Tenía cabellera rubia y larga, lacia, y mi imagen lamía unos pequeñísimos pechos puntiagudos al tiempo que las manos encerraban unas nalgas pequeñas pero perfectamente redondeadas. (2012: 24)

Pero amén de su incontestable carácter lúdico, de estos curiosos desdoblamientos identitarios de la voz narradora y de su personaje, o de su uso, ciertamente acerado, del fantástico y del género policial, quizá sea también importante notar que, atendiendo al trabajo de Ricardo Piglia, la parodia, un mecanismo que podría parecernos a priori arbitrario, no aparece nunca sino como resultado de un "cambio".

Así, según explica el crítico argentino, "hay parodia porque hay un cambio de función [de la literatura]" (2001: 64). Un "cambio de función", continúa, que "depende y está determinado por el cruce entre la literatura y la sociedad", por lo que "se entiende que para analizar la parodia sea necesario reconstruir en primer lugar las condiciones históricas, sociales e ideológicas que hacen posible el cambio que la parodia vendría a expresar" (67). Parece redundante señalar, en esta senda de Piglia, cuál sería ese "cambio".

Por lo demás, cuando hacia el final de la novela Levrero escribe el siguiente pasaje, es obvio que, aunque se guarde de hacerlo a través de una máscara ${ }^{11}$, en su

${ }^{11}$ El mismo año en que se publica Nick Carter se divierte mientras el lector es asesinado y yo agonizo, el célebre semanario Marcha publica un cuento de Nelson Marra titulado "El guardaespaldas" como ganador de un concurso promovido por la revista. Aquel relato, cuenta Mabel Moraña, tuvo la mala fortuna de ser leído por las fuerzas policiales como pornográfico y llamante a la subversión, por lo que además de la brutal experiencia de la 
caso humorística, está mofándose de los discursos militares. Vale decir, está entendiendo la literatura fantástica a la manera de Sylvia Molloy, como ese "género mal llamado de evasión" que posibilita "una vía alternativa" a través de la que poder "expresar nuestra ansiedad" (Molloy, 1991: 107) ante una realidad que, sin duda, a Levrero ya le ha hecho reconfigurar sus cauces y modos:

Soy rápidamente desplazado por una voz que llega desde arriba; apoyado en la barandilla del primer piso, cerca de la habitación de Lady Dunsay, está Tinker. Se ha vestido con ropas militares, oscuras, y en pleno usufructo de la parte más aguda de su reciente estado de euforia, comienza un encendido discurso.

—Damas y caballeros; compatriotas; conciudadanos; pueblo: estoy aquí (aplausos entusiastas del público, que ha comenzado a ponerse de pie, y se va reuniendo bajo las luces, frente al nuevo líder; Tinker extiende las manos, pidiendo silencio con la amable tolerancia de los oradores que, si bien gratificados por el aplauso, quieren seguir hablando). Estoy aquí, decía (aplausos) porque la Patria me reclama (aplausos prolongados). En estos momentos de crisis (aplausos; "muy bien", "muy bien"), y con la serenidad que nos caracteriza (aplausos, asentimientos), es preciso que hagamos llegar nuestra palabra (aplausos, comentarios aprobatorios). Porque nadie debe llamarse a engaño (“¡muy bien!”, aplausos y una pequeña ovación). Las dificultades son muchas (aplausos fervientes) y verdaderas (aplausos más fervientes aún).

Miro el reloj. Según mis cálculos, el estado de euforia le durará por lo menos veinte minutos más. Es insoportable. Sé que luego irá decayendo, pero mientras tanto no puedo tolerarlo. (Levrero, 2012: 63)

\section{A modo de conclusión}

En 1973, en paralelo al estallido de la dictadura, la narrativa de Mario Levrero superó sus espacios de la opresión y la pesadilla, los laberintos donde una puerta conduce a otra puerta y esa puerta a otra puerta y esa puerta a otra puerta en la que alguien escribió "NO HAY SALIDA. ESTO ES EL INFIERNO", las ciudades habitadas por seres con los que será imposible comunicarse o que refieren un extraño u oculto reglamento, los lugares donde desembarcan nazis para rodearnos de soldados y mosquetes. Por primera vez, el humor -utilizado como útil, como auxiliadora terapia levreriana- toma posiciones en su narrativa.

Pero este nuevo modo expresivo de Nick Carter se divierte mientras el lector es asesinado y yo agonizo, que desborda su anterior producción y se levanta en libertad para emerger como un punto de agua entre la tierra amarilla, seca, no puede

tortura para el autor y cuatro años de prisión, supondría el encarcelamiento de, entre otros, Juan Carlos Onetti en tanto que miembro del jurado que lo celebró (Moraña, 1988: 139145). 
explicarse solo como fruto de una imaginación que piruetea, sino que amerita una mirada sociocrítica que la razone y la explique.

Esa muda, considero, es consecuencia directa de una estrenada "autocensura paranoica" propiciada por un contexto que desde ahora le anilla y que -a decir de su hija- terminará por "agravar y cronificar" en él muchas de sus fobias y miedos: la irrupción de lo nefando convierte, de súbito, su escritura de la opresión en 'susceptible' de presentar fisuras por las que podrían caber lecturas críticas no deseadas, análisis de clave alegórica, 'diagnósticos'.

En el insiliado Levrero, en el aparente despegado del afuera, ese irrumpir de época nueva, este nuevo modo narrativo, ese libertinaje, ese desborde, esos procedimientos de escape a lo vitando son a la vez un cementar de fisuras, un repliegue, un resultado.

\section{BIBLIOGRAFÍA}

AÍNSA, Fernando.

2002 Del canon a la periferia. Encuentros y transgresiones en la literatura uruguaya. Montevideo: Trilce.

1993 Nuevas fronteras de la narrativa uruguaya (1960-1993). Montevideo: Trilce.

DE Rosso, Ezequiel (comp.).

2013 La máquina de pensar en Mario. Ensayos sobre la obra de Levrero. Buenos Aires: Eterna Cadencia.

ECHEVARRÍA, Ignacio.

2008a "Levrero y los pájaros", en Pablo Silva Olazábal, Conversaciones con Mario Levrero, pp. 93-102. Montevideo: Trilce.

2008b "Prólogo" a La ciudad, en Mario Levrero, La ciudad, pp. 7-13. Barcelona: Random House Mondadori.

GANDOLFO, Elvio.

2013 Un silencio menos. Conversaciones con Mario Levrero. Buenos Aires: Mansalva.

LEVRERO, Mario.

2012 Nick Carter se divierte mientras el lector es asesinado y yo agonizo y otras novelas. Barcelona: Random House Mondadori.

2008 El lugar. Barcelona: Random House Mondadori. MOLLOY, Sylvia.

1991 "Historia y fantasmagoría", en Enriqueta Morillas Ventura (ed.), El relato fantástico en España e Hispanoamérica, pp. 105-112. Madrid: Sociedad Estatal Quinto Centenario. 
MONTOYA JUÁREZ, Jesús.

2013 Mario Levrero para armar. Jorge Varlotta y el libertinaje imaginativo. Montevideo: Trilce.

MORAÑA, Mabel.

1988 Memorias de la generación fantasma. Montevideo: Monte Sexto.

OLIVERA, Jorge.

2008 Intrusismos de lo real en la narrativa de Mario Levrero. Tesis doctoral. Madrid: Universidad Complutense. Disponible en línea en http://eprints. ucm.es/8631/1/T30796.pdf [Consultado en abril 2014].

PIGLIA, Ricardo.

2001 Crítica y ficción. Barcelona: Anagrama.

RAMA, Ángel.

1972 La generación crítica (1939-1969). Montevideo: Arca.

SARTRE, Jean Paul.

1967 ¿Qué es la literatura? Buenos Aires: Losada.

Silva OlazÁBAL, Pablo.

2008 Conversaciones con Mario Levrero. Montevideo: Trilce. 